\title{
Lumbar paraspinal compartment syndrome
}

\author{
Senthil T. Nathan - Craig S. Roberts • David Deliberato
}

Received: 25 August 2011 / Accepted: 30 September 2011 /Published online: 26 October 2011

(C) Springer-Verlag 2011

\begin{abstract}
The purpose of this study was to systematically review the available evidence on lumbar paraspinal compartment syndrome with specific reference to patient demographics, aetiology, types, diagnosis, clinical features, and treatment. This was an Institutional Review Boardexempt study performed at a Level 1 trauma center. A PubMed search was conducted with the title query: lumbar paraspinal compartment syndrome. Eleven articles met our search criteria. Three of the patients with acute paraspinal compartmental syndrome treated with fasciotomy had a full recovery and were able to resume skiing after four months. The aetiology of the onset of lumbar paraspinal compartment syndrome is broadly divided into acute and chronic. Lumbar paraspinal compartment syndrome is one of the causes of back pain with diagnostic clinical features which should be considered in the differential diagnosis of a patient with low back pain. Prospective multicentre trials may provide the surgeon with more insight into the diagnosis and management of lumbar paraspinal compartment syndrome.
\end{abstract}

\section{Introduction}

von Volkmann [1] first described ischemic muscle necrosis and compartment syndrome in 1881. Since that time, compartment syndromes have been reported in nearly every anatomical area of the extremities (e.g., forearm, calf, thigh, foot, gluteal region, etc.). The lumbar paraspinal region has also been reported to be at risk of compartment syndrome.

S. T. Nathan $\cdot$ C. S. Roberts $(\bowtie) \cdot$ D. Deliberato

Department of Orthopaedic Surgery,

University of Louisville School of Medicine,

210 E. Gray Street, Suite 1003,

Louisville, KY 40202, USA

e-mail: craig.roberts@louisville.edu
To the authors' knowledge, there are no collected data or published clinical series of lumbar paraspinal compartmental syndrome. The purpose of this study was to systematically review the available evidence on lumbar paraspinal compartment syndrome with specific reference to patient demographics, aetiology, types, diagnosis, clinical features, and treatment.

\section{Materials and methods}

This was an Institutional Review Board-exempt study performed at a Level 1 trauma center. A PubMed search was conducted with the title query: lumbar paraspinal compartment syndrome. This search yielded ten articles. Another search of the OVID Medical database was performed with medical subject headings and keywords "lumbar paraspinal compartment syndrome," which yielded nine articles. One author selected high yield abstracts and obtained full articles for the review. Studies selected were original articles that contained one or more case reports of lumbar paraspinal compartment syndrome. Only articles written in English were included. A total of eleven articles met the criteria. Selected articles were analysed for the aetiology, types of lumbar paraspinal compartment syndrome, clinical features, diagnosis, and treatment. Relevant information regarding gender, average age, aetiology of compartment syndrome, types of compartment syndrome, distinctive clinical features, and treatment were carefully extracted.

\section{Results}

Eleven articles met our search criteria, of which nine [2-10] focused on acute lumbar paraspinal compartment syndrome 
in ten patients, and two $[11,12]$ on chronic lumbar paraspinal compartment syndrome in eight patients. Table 1 illustrates a classification system for lumbar paraspinal compartment syndrome. All studies were retrospective case reports; there were no large series or prospective randomised studies. Ten articles reported the age and gender of the patients. Ten of the eleven (91\%) patients were males, and the average age was 39 years (range, 24-67 years) for each type. Table 2 illustrates the demographics of acute lumbar paraspinal compartmental syndrome.

\section{Aetiology}

Our review revealed that acute syndrome was predominantly due to three different aetiologies. Five patients had an atraumatic acute syndrome following either downhill skiing (three patients), $[2,3,5]$ surfboarding (one patient) [4], or weight lifting (one patient) [4]. One patient had a direct injury to the paraspinal muscle [9] and three patients developed acute syndrome following non-spinal surgery such as aortic bypass and gastric bypass [6-8]. One patient was reported to have abused isotretoinoin, cocaine, and testosterone [10]. One patient who developed chronic syndrome was a professional weight lifter [12]. One case report on chronic syndrome did not comment on the aetiology of the chronic syndrome [11].

\section{Diagnosis}

\section{Clinical features}

All patients with acute lumbar paraspinal compartment syndrome had distinctive clinical features to establish a clinical diagnosis (Table 3 ). All presented with excruciating low back pain with or without radiation within 12 hours of the incident $[2-4,6,8]$, but without prior history of such pain. On examination, all patients had localised tenderness over the paraspinal region with a board-like rigidity of the paraspinal region. Abdominal sounds were absent or diminished in all, with or without deep abdominal tenderness on palpation. Localised loss of sensation over the paraspinal region was found in all patients and was considered as pathognomonic of the acute syndrome. Only one patient [3] had evidence of motor involvement in the form of atrophy of the abdominal and paraspinal muscles. None of the patients had any form of neurological abnormality in the legs. Diagnostic criteria for acute lumbar paraspinal compartment syndrome are shown in Table 4.

Patients with chronic exertional lumbar paraspinal compartmental syndrome had certain features which differentiated them from the acute syndrome patients (Table 5). These patients typically had no symptoms at rest; however, they experienced severe back pain on exertion which was relieved with extension but made worse with flexion. These patients had no history of previous spinal trauma or localised neurological manifestations. All had normal range of motion of the spine. None of the reports on chronic lumbar paraspinal compartment syndrome delineated a specific time frame for the onset of symptoms; however, patients experienced back pain on repetitive exertion.

\section{Laboratory findings}

Patients with acute lumbar paraspinal compartment syndrome presented with evidence of rhabdomyolysis. Creatinine phosphokinase was elevated to above 5000 international units/liter (IU/L) (range, 5000-82,000), serum glutamic-oxaloacetic transaminase (SGOT) was elevated (mean, $150 \mathrm{IU} / \mathrm{L}$ ), and serum and urine myoglobin was reported in all patients. Three patients $(30 \%)$ had progressive elevation of creatine phosphokinase (CPK) and myoglobin over 48-72 hours after the onset of pain, whilst in seven patients these parameters gradually decreased over 48-72 hours. In six patients who had magnetic resonance imaging (MRI) of the paraspinal region, homogenous signal changes in T2-weighted image resembling paraspinal oedema were noted. Intramuscular compartmental pressure (IMR) was measured in three of the ten patients. In seven patients, IMR was not done at the time of initial evaluation because the compartment syndrome was not recognised as a possible cause of the symptoms. The IMR range was 70 $80 \mathrm{~mm} \mathrm{Hg}$ (normal, 3.1-7 mm Hg).

Patients with chronic exertional compartment syndrome had normal blood studies. However, the intramuscular
Table 1 Classification of lumbar paraspinal compartment syndrome

\begin{tabular}{ll}
\hline Classification type & Description \\
\hline Type I & Acute lumbar paraspinal compartment syndrome \\
Subtype A & Atraumatic acute compartment syndrome — downhill skiing, surf boarding, \\
& weight lifting \\
Subtype B & Direct trauma to the paraspinal muscles \\
Subtype C & Secondary to non-spinal surgery—aortic bypass and gastric bypass surgery \\
Type II & Chronic exertional lumbar paraspinal compartment syndrome \\
\hline
\end{tabular}


Table 2 Demographics of acute lumbar paraspinal compartment syndrome
*Type I (A, B, C);

Type II - Based on classification of lumbar paraspinal compartment syndrome

\begin{tabular}{lllllll}
\hline Author & Year & Number of patients & Sex & Age & Aetiology & Type* \\
\hline Carr et al. [2] & 1985 & 1 & Male & 24 & Downhill skiing & I-A \\
DiFazio et al. [5] & 1991 & 1 & Male & 27 & Downhill skiing & I-A \\
Sava et al. [9] & 1999 & 1 & Female & 43 & Direct trauma & I-B \\
Osamura et al. [7] & 2000 & 1 & Male & 67 & Aortic bypass & I-C \\
Kitajima et al. [4] & 2002 & 1 & Male & 25 & Surfboarding & I-A \\
Ferreira et al. [6] & 2003 & 1 & Male & 55 & Aortic bypass & I-C \\
Khan et al. [3] & 2005 & 1 & Male & 35 & Downhill skiing & I-A \\
Haig et al. [8] & 2009 & 2 & Male & 57 & Aortic bypass & I-C \\
& & & Male & 34 & Gastric bypass & I-C \\
Wik et al. [10] & 2010 & 1 & Male & 30 & Weight lifting & I-A \\
\hline
\end{tabular}

compartment pressure was elevated in all eight patients and ranged from 70-80 $\mathrm{mm} \mathrm{Hg}$. Konno et al. [11] reported that seven of eight patients with chronic exertional lumbar paraspinal compartment syndrome had a typical waxing and plateau pattern of pressure changes with the onset of back pain.

\section{Treatment}

The initial management in all patients with acute syndrome was aimed to control pain and rhabdomyolysis by administration of crystalloid fluid infusions, analgesics, urine alkalisation, and bed rest. Three of these patients (30\%) [3, $4,7]$ who continued to develop severe rhabdomyolysis after 48-72 hours underwent fasciotomy. All patients with chronic syndrome underwent fasciotomy.

\section{Outcomes}

Three of the patients with acute paraspinal compartmental syndrome treated with fasciotomy had a full recovery and were able to resume skiing after four months [3, 4, 7]. The seven patients who were treated conservatively resumed their physical activity after six months, but with mild chronic back pain on vigorous exertion [5, 8-10]. Patients who underwent fasciotomy for chronic paraspinal syndrome resumed their work activities without any difficulty after four to six months.

\section{Discussion}

The aetiology of the onset of lumbar paraspinal compartment syndrome is broadly divided into acute and chronic. Among the ten patients with acute lumbar paraspinal compartment syndrome, five had an atraumatic acute syndrome (Type I-A) following downhill skiing (three patients) [2, 3, 5], surfboarding (one patient) [4], or unconditioned weight lifting (one patient) [4]. One patient had a direct injury to the paraspinal muscle (Type I-B) [9] and three patients developed acute syndrome following non-spinal surgery such as aortic bypass and gastric bypass

Table 3 Diagnosis of acute lumbar paraspinal compartment syndrome

\begin{tabular}{|c|c|c|c|c|c|c|c|}
\hline Author & $\begin{array}{l}\text { Back } \\
\text { pain }\end{array}$ & $\begin{array}{l}\text { Localised sensory loss } \\
\text { paraspinal region }\end{array}$ & $\begin{array}{l}\text { Absent bowel } \\
\text { sounds }\end{array}$ & Motor symptoms & $\begin{array}{l}\text { Intramuscular } \\
\text { pressure }(\mathrm{mm} \mathrm{Hg})\end{array}$ & $\begin{array}{l}\text { CPK } \\
(\mathrm{IU} / \mathrm{L})\end{array}$ & Treatment \\
\hline Carr et al. [2] & + & + & + & - & - & 5,465 & Conservative \\
\hline DiFazio et al. [5] & + & + & + & - & & 60,000 & Conservative \\
\hline Sava et al. [9] & + & + & + & - & - & 10,581 & Conservative \\
\hline Osamura et al. [7] & + & + & + & - & - & 7,975 & Fasciotomy \\
\hline Kitajima et al. [4] & + & + & + & - & $14-16$ & 21,440 & Fasciotomy \\
\hline Ferreira et al. [6] & + & + & + & - & - & 25,000 & Conservative \\
\hline Khan et al. [3] & + & + & + & $\begin{array}{l}\text { Atrophy of } \\
\text { paraspinal muscle }\end{array}$ & $\begin{array}{l}44(\mathrm{R}) \\
26(\mathrm{~L})\end{array}$ & 59,863 & Fasciotomy \\
\hline Haig et al. [8] & + & + & + & - & - & - & Conservative \\
\hline Wik et al. [10] & + & + & + & - & $\begin{array}{l}150(\mathrm{R}) \\
20(\mathrm{~L})\end{array}$ & 82,000 & Conservative \\
\hline
\end{tabular}

$R$ right paraspinal muscular compartment, $L$ left paraspinal muscle compartment, $C P K$ creatinine phosphokinase 
Table 4 Diagnostic criteria for acute lumbar paraspinal compartment syndrome
Criteria/Description

1. Excruciating localised back pain following an inciting event such as downhill skiing, direct trauma or post-surgery. Pain increased on flexion and relieved on extension

2. No prior history of back pain

3. Clinical features:

(i) Bilateral or unilateral paraspinal muscle board like rigidity

(ii) Hypoactive bowel sounds with diffuse abdominal tenderness

(iii) Localised sensory loss in the paraspinal region with or without localised muscleatrophy

4. Investigations:

(i) Elevated CPK (more than 2,000-40,000)

(ii) Elevated SGOT

(iii) Urine myoglobinuria

(iv) Elevated intramuscular pressure - approximately $70-80 \mathrm{~mm} \mathrm{Hg}$

(v) MRI - Homogenous increase in signal intensity in T2 within the compartment
(Type I-C). The chronic lumbar paraspinal compartment syndromes were caused by chronic exertion (Type II).

Cadaveric dissections of the lumbar region clearly identify a well-developed compartment of posterior spinal muscles encased by the posterior and middle layer of the lumbodorsal fascia $[2,13]$. The posterior layer divides the intrinsic (paraspinal) from the extrinsic (upper limb and serratus posterior) muscles of the back. The posterior layer is composed of two clearly distinct superficial and deep layers that encompass the iliocostalis, longissimus, and multifidus muscles [2, 13, 14]. Fascial continuity of the posterior layer exists with the latissimus dorsi superiorly with that of the superficial layer with the fascia in the dorsal spine, and inferiorly with its extensive attachments to the gluteus medius and maximus and the sacrum and ilium. Based on the fascial continuity, it appears that the posterior layer acts as an intermediary for transmission of mechanical forces between the upper and lower limbs, the right and left sides of the body, and the abdominal walls and spine [15]. Peck et al. [16] demonstrated the connections of the

Table 5 Diagnostic criteria for chronic lumbar paraspinal compartment syndrome

\section{Diagnostic criteria}

1. No symptoms at rest. Pain on exertion such as exercise

2. No prior history trauma to the spine

3. Clinical features:

(i) Normal range of motion

(ii) Improvement of pain on extension during exertion

(iii) No neurological deficits

4. Investigations:

(i) Elevated intramuscular pressure and muscle relaxation pressure

(ii) Normal CPK, SGOT

(iii) Negative MRI posterior layer from the occiput to the sacrum by injecting dye and staining the paraspinal muscles including the multifidus, splenius cervicis, and capitis muscles.

Muscle traction studies by Bogduk and MacIntosh [17] described the presence of "posterior accessory ligaments" which are bands of thick fascia in the deep layer that pass laterally from the lumbar spinous process between L2 and L5 to the posterior superior iliac spine and lateral raphe, lying posterior to the quadratus lumborum muscle. It has been postulated [13] that these accessory ligaments may semi-compartmentalise the paraspinal muscles, particularly the multifidus, which increases the chances of development of unilateral or bilateral paraspinal compartment syndrome in response to an insult. Vleeming et al. [15] described the "foreclosure of sacroiliac joint", which is a displacement of the deep layer up to L5-S1 as load transfer occurs across the sacrotuberous ligaments, leading to increased pressure within the compartment.

The posterior layer of the lumbodorsal fascia resists maximum flexion movements of the spine [2]. Three different mechanisms are hypothesised by which the posterior layer resists these moments. The first is by maintaining a passive tension in the posterior ligamentous system including the posterior layer $[18,19]$. The second mechanism involves the obliquely-directed fibres of the posterior layer which may tighten on contraction of the attaching muscles such as the transverse abdominis, internal oblique, latissimus dorsi, and gluteus maximus [18-22]. Based on cadaveric studies [17-21], the orientation of the superolateral fibres is more vertical than the inferolateral fibres. This creates an asymmetric lattice within the superficial lamellae of the posterior layer which may increase the maximum extensor moment generated within this layer from contraction of the paraspinal muscles that occurs during downhill skiing or surfboarding. The third mechanism involves muscle contraction. Gracovetsky et al. 
[18] suggested that by tightly enclosing the paraspinal muscle in a compartment, the posterior layer increases the effectiveness of the contraction by limiting radial expansion, termed "the hydraulic amplifier effect." Hukins et al. [23] indicated that the thoracolumbar fascia may increase the effectiveness of the paraspinal muscles by approximately $17-30 \%$. This finding is supported by the anatomical and physiological studies of Carr et al. [2] which show that a slow increase in the effectiveness with sustained contraction of the paraspinal muscles leads to development of compartment syndrome. A similar type of strain occurs during lifting heavy weights.

The thoracolumbar fascia is able to alter its thickness based on the stresses incurred [24]. The posterior layer of the fascia has been reported to stiffen (resist deformation) with successive loading [24]. In the leg, fascial thickening is associated with chronic compartment syndrome in response to loading $[25,26]$. According to Farfan et al. [27], adaptive fascial thickening has been noted in the posterior layer based on comparative anatomical observations with other anthropoids. This finding forms the basis for various exercise regimens to treat low back pain by adaptive strengthening of the posterior layer and muscles.

The use of isoretinoin and cocaine was reported in one patient with acute lumbar paraspinal compartmental syndrome [10]. There have been case reports of muscle damage and rhabdomyolysis after the intake of isotretinoin $[10,22,28,29]$. Other agents that have been associated with muscle damage include excessive alcohol consumption, intramuscular injection of anabolic steroids, and cocaine $[10,30,31]$. There is no clear association between substance abuse and the development of lumbar paraspinal compartment syndrome.

The pathophysiological mechanism involved in the development of lumbar paraspinal compartment syndrome during aortic bypass and gastric bypass may be due to hypoperfusion or occlusion of the lumbar arteries from aortic clamping and ligation of the aneurysmal sac. This ischaemic insult may lead to swelling and necrosis of the paraspinal muscle that subsequently increases the compartment pressure, further leading to rhabdomyolysis from compartment syndrome and also to reperfusion injury that occurs upon clamp removal.

All patients with acute lumbar paraspinal compartment had specific features that differentiated this diagnosis from other causes of back pain. All patients had superficial paraspinal sensory loss and absent bowel sounds. This combined with intact neurological signs may be pathognomonic in the early diagnosis of this syndrome. A disc herniation could cause paraspinal sensory loss, but substantial overlap of multiple levels may not be possible with a single level lesion. In addition, in the context of multiple level involvement of the spine as in spinal stenosis, isolated paraspinal sensory loss is highly unlikely as it is usually associated with sensory loss in the legs. An apparent entrapment syndrome of the lumbar dorsal ramus or a selective dorsal neurotomy does not affect the sensation of the paraspinal region. Absent bowel sounds may be due to the development of paralytic ilieus due to a biochemical abnormality from rhabdomyolysis. Table 4 illustrates the diagnostic criteria for the diagnosis of acute lumbar paraspinal compartmental syndrome.

Patients with chronic lumbar paraspinal compartment syndrome had typical features of exaggerated low back pain on exertion. They were asymptomatic at rest and had a normal range of motion of the spine. All patients had normal neurology on initial examination. Table 5 illustrates the diagnostic criteria for the diagnosis of chronic lumbar paraspinal compartment syndrome.

Although compartment syndrome has many aetiologies, the underlying basic pathology is an elevated intracompartmental pressure. Measurement of a baseline intramuscular pressure of the paraspinal compartment is an objective technique of diagnosing compartment syndrome $[2,11,32$, 33]. The intramuscular pressure can be measured using a microtip fibre optic catheter transducer [11,34] or a micro capillary infusion method [32, 33, 35]. The resting pressure varies considerably based on the posture and valsalva pressure [2]. Songcharoen et al. [35] measured the baseline resting intramuscular pressure as $3.11 \mathrm{mg} \mathrm{Hg}$ in the prone position, $7.95 \mathrm{~mm} \mathrm{Hg}$ in the sitting position, $5.21 \mathrm{~mm} \mathrm{Hg}$ at rest, and $4.47 \mathrm{~mm} \mathrm{Hg}$ in the standing position which were comparable to pressures in the other muscular compartments in the body $[17,35]$. The increase in intramuscular pressure during sitting corresponds to the increase in intradiscal pressure as described by Nachemson et al. [36]. The paraspinal compartmental pressure was found to be only slightly elevated on maximum flexion [35]. The explanation for this decreased or no compartmental pressure in this position was explained by Carr et al. [2] as the spine hangs from its ligaments or by other muscle groups on forward flexion. In patients with both acute and chronic lumbar paraspinal compartment syndrome, the intramuscular compartmental pressure was found to range from 70 to $150 \mathrm{~mm} \mathrm{Hg}$.

Konno et al. [11] described four different patterns of intramuscular pressure changes in patients associated with back pain. Patients with clinical suspicion of lumbar compartmental syndrome demonstrated a waxing pattern (pressure increased at the onset of back pain and continued to increase thereafter) or a plateau pattern (pressure increased when back pain developed and remained constant) of intramuscular pressure changes within the erector spinae with the onset of back pain. In addition, spinal alignment changed from lordosis to kyphosis, which increased the intramuscular pressure and decreased the blood flow [11]. 
Measurement of intramuscular pressure in the erector spinae during and after isometric and concentric exercise, as well as estimating the muscle relaxation pressure, is more relevant in the diagnosis of chronic lumbar paraspinal compartment syndrome. Physiological studies [32] have shown an increase in the volume of skeletal muscle up to $20 \%$ during heavy exercise. It has also been demonstrated that the muscle relaxation pressure is directly proportional to the volume load in other muscular compartments [32, 33]. Styf [32, 33] reported the mean muscle contraction pressure of the paraspinal compartment was $175 \mathrm{~mm} \mathrm{Hg}$. The relaxation pressure increased from 8.5 to $14 \mathrm{~mm} \mathrm{Hg}$ during an exercise stress test and the intramuscular pressure fell to pre-existing levels before exercise within six minutes. Thus, the measurement of muscle relaxation pressure during exercise and intramuscular pressure after exercise is useful in the diagnosis of exercise-induced chronic lumbar paraspinal compartmental syndrome.

MRI findings in acute lumbar paraspinal compartment syndrome were striking and uniform in the six cases reported [3, 7-10]. All patients had increased signal intensity on T2 weighted images, mimicking the presence of paraspinal oedema. Follow-up MRI in all patients showed some form of atrophy of the paraspinal muscles when compared with the other musculature. This atrophy was reported to be more evident in patients who had conservative management for acute syndrome.

Based on the available evidence and the trend towards good functional recovery following fasciotomy, it appears that all patients should be treated surgically. Patients treated conservatively returned to baseline function within four to six months; however, they tended to have chronic back pain on exertional activities. Even so, initial conservative measures should include administration of crystalloid fluid infusions, analgesics, urine alkalisation, and bed rest to avoid the development of full blown rhadomyolysis and acute renal failure.

Our study represents the first systematic review of lumbar paraspinal compartmental syndrome. Even though the review is based on existing evidence of paraspinal compartment syndrome, this opens an array of questions on patients with low back pain with no anatomical abnormality in whom measurement of intramuscular compartmental pressure may be detrimental in identifying the cause of pain. Our study is limited to a small number of patients available in the literature.

\section{Conclusion}

Lumbar paraspinal compartment syndrome is one of the causes of back pain with diagnostic clinical features which should be considered in the differential diagnosis of a patient with low back pain. A thorough clinical examination and MRI aid in the diagnosis. Measurement of paraspinal compartment pressure is an option when clinical and imaging evaluations are inconclusive. Conservative measures are the first line of management and include opioids for pain control and treatment of rhabdomyolysis. As in other muscular compartments of the body, the basic trend is to surgically decompress the compartment as early as possible to avoid long-term functional sequelae. Prospective multicentre trials with clear delineation of the types may provide more insight into the diagnosis and management of lumbar paraspinal compartment syndrome.

\section{References}

1. von Volkmann R (1881) Die ischämischen Muskellähmungen und Kontracturen. Centralblatt für Chirurgie, Leipzig 8:801-803

2. Carr D, Gilbertson L, Frymoyer J, Krag M, Pope M (1985) Lumbar paraspinal compartment syndrome. A case report with physiologic and anatomic studies. Spine (Phila $\mathrm{Pa}$ 1976) $10: 816-820$

3. Khan RJ, Fick DP, Guier CA, Menolascino MJ, Neal MC (2005) Acute paraspinal compartment syndrome. A case report. J Bone Joint Surg Am 87:1126-1128

4. Kitajima I, Tachibana S, Hirota Y, Nakamichi K (2002) Acute paraspinal muscle compartment syndrome treated with surgical decompression: a case report. Am J Sports Med 30:283-285

5. DiFazio FA, Barth RA, Frymoyer JW (1991) Acute lumbar paraspinal compartment syndrome. A case report. J Bone Joint Surg Am 73:1101-1103

6. Ferreira J, Galle C, Aminian A, Michel P, Guyot S, De Wilde JP et al (2003) Lumbar paraspinal rhabdomyolysis and compartment syndrome after abdominal aortic aneurysm repair. J Vasc Surg 37:198-201

7. Osamura N, Takahashi K, Endo M, Kurumaya H, Shima I (2000) Lumbar paraspinal myonecrosis after abdominal vascular surgery: a case report. Spine (Phila Pa 1976) 25:1852-1854

8. Haig AJ, Hartigan AG, Quint D (2009) Low back pain after nonspinal surgery: the characteristics of presumed lumbar paraspinal compartment syndrome. PM R 1:383-388

9. Sava J, Moelleken A, Waxman K (1999) Cardiac arrest caused by reperfusion injury after lumbar paraspinal compartment syndrome. J Trauma 46:196-197

10. Wik L, Patterson JM, Oswald AE (2010) Exertional paraspinal muscle rhabdomyolysis and compartment syndrome: a cause of back pain not to be missed. Clin Rheumatol 29:803-805

11. Konno S, Kikuchi S, Nagaosa Y (1994) The relationship between intramuscular pressure of the paraspinal muscles and low back pain. Spine (Phila Pa 1976) 19:2186-2189

12. Xu YM, Bai YH, Li QT, Yu H, Cao ML (2009) Chronic lumbar paraspinal compartment syndrome: a case report and review of the literature. J Bone Joint Surg Br 91:1628-1630

13. Barker PJ, Briggs CA (1999) Attachments of the posterior layer of lumbar fascia. Spine (Phila Pa 1976) 24:1757-1764

14. Moore KL, Dalley AF (1999) Clinically oriented anatomy. Williams and Wilkins, New York, pp 503-663

15. Vleeming A, Pool-Goudzwaard AL, Stoeckart R, van Wingerden JP, Snijders CJ (1995) The posterior layer of the thoracolumbar fascia. Its function in load transfer from spine to legs. Spine (Phila Pa 1976) 20:753-758 
16. Peck D, Nicholls PJ, Beard C, Allen JR (1986) Are there compartment syndromes in some patients with idiopathic back pain? Spine (Phila Pa 1976) 11:468-475

17. Bogduk N, Macintosh JE (1984) The applied anatomy of the thoracolumbar fascia. Spine (Phila Pa 1976) 9:164-170

18. Gracovetsky S, Farfan HF, Lamy C (1977) A mathematical model of the lumbar spine using an optimized system to control muscles and ligaments. Orthop Clin North Am 8:135-153

19. Gracovetsky S, Farfan H, Helleur C (1985) The abdominal mechanism. Spine (Phila Pa 1976) 10:317-324

20. Fairbank JC, O'Brien JP (1980) The abdominal cavity and thoracolumbar fascia as stabilizers of the lumbar spine in patients with low back pain: Engineering aspects of the spine. Medical Engineering Publications, London, pp 83-88

21. McGill SM, Norman RW (1988) Potential of lumbodorsal fascia forces to generate back extension moments during squat lifts. J Biomed Eng 10:312-318

22. Tesh KM, Dunn JS, Evans JH (1987) The abdominal muscles and vertebral stability. Spine (Phila Pa 1976) 12:501-508

23. Hukins DL, Aspden RM, Hickey DS (1990) Thorecolumlbar fascia can increase the efficiency of the erector spinae muscles. Clin Biomech (Bristol, Avon) 5:30-34

24. Yahia LH, Pigeon P, DesRosiers EA (1993) Viscoelastic properties of the human lumbodorsal fascia. J Biomed Eng 15:425-429

25. Detmer DE, Sharpe K, Sufit RL, Girdley FM (1985) Chronic compartment syndrome: diagnosis, management, and outcomes. Am J Sports Med 13:162-170

26. Turnipseed WD, Hurschler C, Vanderby R Jr (1995) The effects of elevated compartment pressure on tibial arteriovenous flow and relationship of mechanical and biochemical characteristics of fascia to genesis of chronic anterior compartment syndrome. J Vasc Surg 21:810-816, discussion 816-817

27. Farfan HF (1975) Muscular mechanism of the lumbar spine and the position of power and efficiency. Orthop Clin North Am $6: 135-144$

28. Hodak E, Gadoth N, David M, Sandbank M (1986) Muscle damage induced by isotretinoin. Br Med J (Clin Res Ed) 293:425-426

29. Tillman DM, White SI, Aitchinson TC (1990) Isotretinoin, creatinine kinase and exercise. Br J Dermatol 123:22-23

30. Farkash U, Shabshin N, Pritsch Perry M (2009) Rhabdomyolysis of the deltoid muscle in a bodybuilder using anabolic-androgenic steroids: a case report. J Athl Train 44:98-100

31. Singhal P, Horowitz B, Quinones MC, Sommer M, Faulkner M, Grosser M (1989) Acute renal failure following cocaine abuse. Nephron 52:76-78

32. Styf J (1987) Pressure in the erector spinae muscle during exercise. Spine (Phila Pa 1976) 12:675-679

33. Styf J, Lysell E (1987) Chronic compartment syndrome in the erector spinae muscle. Spine (Phila Pa 1976) 12:680-682

34. Crenshaw AG, Styf JR, Mubarak SJ, Hargens AR (1990) A new "transducer-tipped" fiber optic catheter for measuring intramuscular pressures. J Orthop Res 8:464-468

35. Songcharoen P, Chotigavanich C, Thanapipatsiri S (1994) Lumbar paraspinal compartment pressure in back muscle exercise. J Spinal Disord 7:49-53

36. Nachemson AL (1981) Disc pressure measurements. Spine (Phila Pa 1976) 6:93-97 